\title{
Bioinformatics approach for data management about bone cells grown on substitute materials
}

\author{
Federica Viti ${ }^{\otimes}$, Ivan Merelli', Silvia Scaglione ${ }^{2}$, Luciano Milanesi' \\ 'Institute for Biomedical Technologies, National Council of Research, Segrate, Italy \\ ${ }^{2}$ Institute of Electronics Computer and Telecommunication Engineering, National Council of Research, Genoa, Italy
}

\section{Motivation and Objectives}

Tissue engineering, the research field aimed at finding high technological biomaterials able to restore, maintain, or improve tissue function, concentrates many efforts in the contest of bone and cartilage, due to their possible wide-spread clinical applications. The main target is the design of well performing scaffolds suitable to promote the development of natural tissue in implant conditions, without generating rejection and, hopefully, degrading in vivo at the same rate of tissue formation.

Concerning both bone and cartilage, one of the most important research aspects is determining the biochemical and topological factors that induce cell differentiation and tissue ingrowth. The scaffold material composition is crucial and many of them have been already tested, including alginate (Duggal et al., 2009), collagen/chitosan (Ravindran et al., 2012), polycaprolactone $(\mathrm{PCL})$ and hydroxyapatite (HA) (Scaglione et al., 2010), Poly-L-Lactide Acid (PLLA) (Ciapetti et al., 2012), polymethylmethacrylate (BombonatoPrado et al., 2007), bioactive glasses (Leven et al., 2004), carbon nanotubes (Van der Zande et al., 2004), etc.

To better evaluate material performance, the biomolecular characterization of the cellular response is becoming a common practice among researchers. Nonetheless, experimental data usually remain sparse in literature: the collection of high-throughput gene expression profiles from samples on different materials could allow data comparisons and formulation of new hypotheses about the effectiveness of bone/cartilage substitute.

In this context authors extended the existing OsteoChondroDB database (Viti et al., 2012), which collects data and metadata from microarray gene expression of cells cultured in different conditions onto diverse materials, and allows analyzing differentially expressed genes (DEG) from the available knowledge base.

\section{Methods}

The OsteoChondroDB relies on MySQL database, while the web interface has been developed using php and javascript technologies, and this improved version of the systems is based on the same infrastructure.

Manual research has been performed on papers containing biomolecular data about osteochondral tissue developed on different scaffolds. Data have been retrieved from known public repositories such as Gene Expression Omnibus (http://www.ncbi.nlm.nih.gov/geo/) and ArrayExpress (http://www.ebi.ac.uk/arrayexpress/), whenever experiments were available, or directly contacting papers authors. The amount of data produced in this field is new, scarce, and variegated, although the importance of biomolecular aspects related to the tissue growth on materials can be of great importance to design improved scaffolds.

To exploit the available data in an integrated fashion, the strategy described by Kodama et al. (Kodama et al., 2012) appears useful, because it proposes a multi-species and multi-platform approach for gene expression microarray data meta-analysis. In this way, it is possible to increase the number of evidences, by considering mouse and rat data together with human experiments and by mixing different versions, brands and designs of microarray chips. The information mapping between species can be performed through AILUN system (Chen R et al., 2007), which converts ids of different platforms.

\section{Results and discussion}

A new section of the OsteoChondroDB (freely accessible to users at url: http://www.itb.cnr.it/ osteochondrogene/) has been created to maintain the collected information. The database is organized into tables containing the considered biomaterial types, the organism and the exploited cells, the array platforms, the PubMed identifier of the paper, some notes about the experi- 
ment and the obtained results. Contextually, the database web interface has been extended to suitably visualize this new information. Collected experiments have been grouped according to the reference biomaterial, and metadata and data about experimental conditions can be browsed from the related section. Data are stored in the file-system and accessed through a link from the database web interface, while metadata are hosted in the database itself. Data retrieval is possible starting from array type, organism and biomaterial.

In order to infer new knowledge about mechanisms involved in bone and cartilage generation, a suitable pipeline (Figure 1) has been designed to mine knowledge from collected information. Microarray data, retrieved from different sources, are maintained into a single database table, whose main fields are the name of the gene, and the expression value in each experiment. Samples are grouped into treatments (diverse biomaterials) and controls, and inter-microarray normalization is performed on the basis of recognized housekeeping genes (de Jonge et al., 2007) that are preserved in each microarray platform. The core of the strategy relies on the approach implemented by Kodama et al.: considering collected datasets as belonging to a single experiment, it produces an integrated model of genes expression values from which a selection of DEG can be statistically inferred.

Gene Ontology (GO) and Kyoto Encyclopedia of Genes and Genomes (KEGG) ontology have been exploited to annotate genes, and define the functional enrichment of the results set (on the basis of the hypergeometric distribution), in order to elucidate the mostly activated functional mechanisms involved in bone tissue generation according to each biomaterial.

Moreover, the database interface has been extended to perform data annotation. The OsteoChondroDB maintains many references related to bone and cartilage that can be easily exploited to annotate DEG with the collected knowledge base. A suitable interface has been developed to upload microarray results, to automatically perform annotation and to retrieve the list enriched by one or more literature references for each DEG.

The OsteoChondroDB improvements discussed in this work offer users the possibility to access data that previously were sparse in literature, providing the possibility of statistically analyzing them in an integrated fashion. Two main features contribute to add value to the application: the automatic annotation of osteochondro

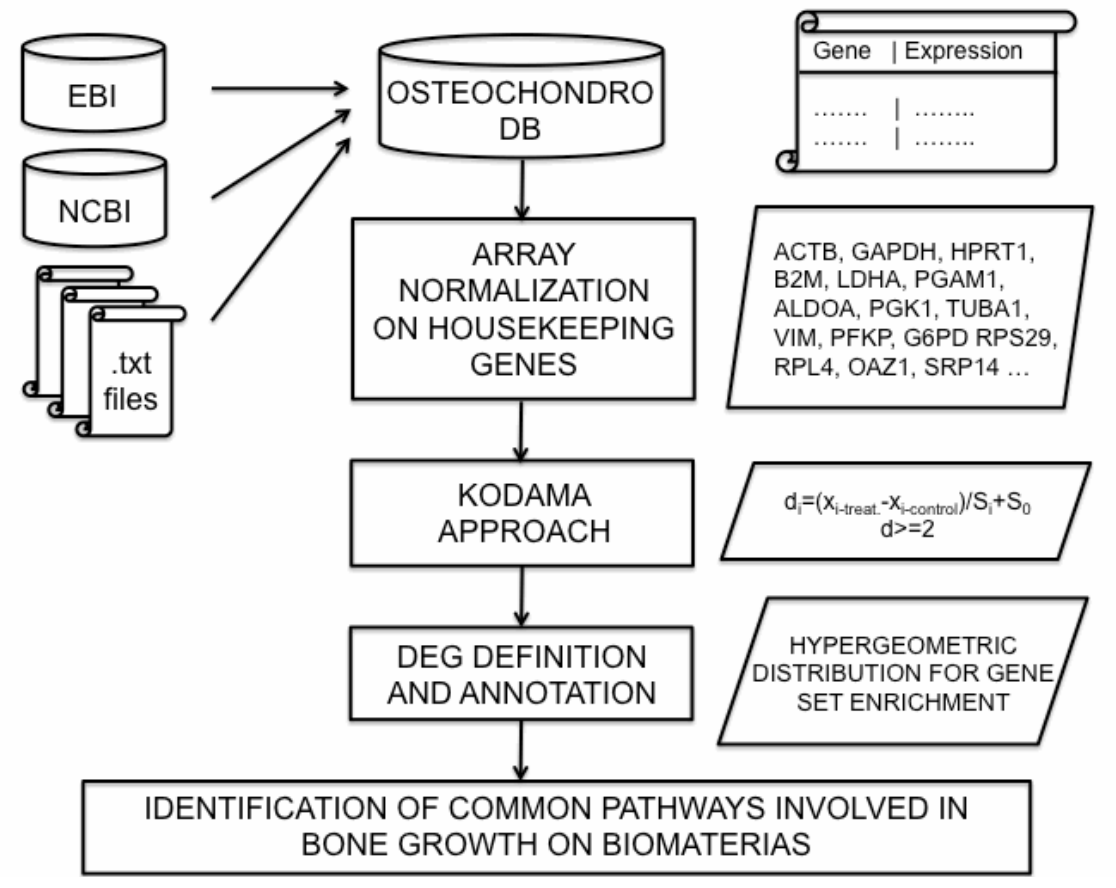

Figure 1: Schema of the designed analysis pipeline. 
genes according to literature references, which improves the information about physiological pathways involved in the development of these tissues, and the exploitation of many integrated microarray data to statistically analyze bone and cartilage ingrowth, that represents a useful support for tissue engineering applications.

\section{Acknowledgements}

This work has been supported by the Italian Ministry Education and Research (MIUR) through the Flagship "InterOmics", ITALBIONET (RBPRO5ZK2Z), HIRMA (RBAPI1YS7K) and the European "MIMOMICS" projects.

\section{References}

1. Bombonato-Prado KF, Bellesini LS, et al. (2007) Microarraybased gene expression analysis of human osteoblasts in response to different biomaterials, J Biomed Mater Res A 88(2), 401-8.

2. Chen R., Li L., and Butte AJ (2007) AlLUN: Reannotating Gene Expression Data Automatically, Nature Methods 4(11), 879

3. Ciapetti G, Granchi D, et al. (2012) Enhancing Osteoconduction of PLLA-Based Nanocomposite Scaffolds for Bone Regeneration Using Different Biomimetic Signals to MSCs, Int. J. Mol. Sci. 13, 24392458; doi:10.3390/ijmst3. 3022439.

4. de Jonge HJM, Fehrmann RSN et al. (2007) Evidence Based Selection of Housekeeping Genes, PLOSONE 2(9), e898, doi:10.1371/journal.pone.0000898
5. Duggal S, Fronsdal KB, et al. (2009) Phenotype and Gene Expression of Human Mesenchymal Stem Cells in Alginate Scaffolds, Tissue Engineering: Part A 15(7). doi: $10.1089=$ ten tea 2008.0306

6. Kodama K, Horikoshi M et al. (2012) Expression-based genome-wide association study links the receptor CD44 in adipose tissue with type 2 diabetes, Proc Natl Acad Sci U S A 109(18), 7049-54

7. Leven RM, Virdi AS et al. (2004) Patterns of gene expression in rat bone marrow stromal cells cultured on titanium alloy discs of different roughness, J Biomed Mater Res A 70(3), 391-401

8. Ravindran S, Gao Q, et al. (2012) Biomimetic Extracellular Matrix-Incorporated Scaffold Induces Osteogenic Gene Expression in Human Marrow Stromal Cells, Tissue Engineering: Part A 18(3-4), doi: 10.1089/ten. tea. 2011.0136

9. Scaglione S, Lazzarini E, llengo C, Quarto R. (2010) A composite material model for improved bone formation. J Tissue Eng Regen Med. 4(7):505-13.

10. Van der Zande M, Walboomers F, et al. (2010) Genetic profiling of osteoblast-like cells cultured on a novel bone reconstructive material, consisting of poly-L-lactide, carbon nanotubes and microhydroxyapatite, in the presence of bone morphogenetic protein-2, Acta Biomater. 6(11),4352-60.

11. Viti F, Merelli I and Milanesi L (2012) OsteoChondroDB: a database about biomolecular chondral-bone development in physiological and diseased conditions, EMBnet. journal 18 